\title{
Microbial synthesis of magnetite nanoparticles for arsenic removal
}

\author{
Gopal Samy Balakrishnan ${ }^{1,3^{*}}$, Karthik Rajendran ${ }^{1}$, Jegatheesan Kalirajan ${ }^{2}$ \\ ${ }^{1}$ Department of biotechnology, Liatris Biosciences LLP, Kottayam, India. \\ ${ }^{2}$ Center for Research and PG Studies in Botany and Department of Biotechnology, Thiagarajar College (Autonomous), Madurai, India. \\ ${ }^{3}$ Department of Biotechnology, Roever College of Engineering and Technology, Perambalur, India.
}

\begin{tabular}{l}
\hline ARTICLE INFO \\
\hline Article history: \\
Received on: December 14, 2019 \\
Accepted on: February 22, 2020 \\
Available online: May 26, 2020 \\
\hline
\end{tabular}

Key words:

Fusarium oxysporum,

nanomagnetite, arsenic,

bioremediation

\begin{tabular}{l}
\hline ABSTRACT \\
\hline Apart from their vast applications, the magnetic property of magnetite nanoparticles make it as opt candidate \\
for the removal of arsenic from drinking water and other polluted water streams. Magnetite nanoparticles \\
were produced by microbial synthesis from Fusarium oxysporum with Ferric Chloride and magnetite ore as \\
substrates. The structure and morphology of magnetite nanoparticles were characterized by Fourier Transform \\
Infrared (FTIR) Spectroscopy, UV-Vis spectroscopy, X-ray Diffraction (XRD), Scanning Electron Microscope \\
(SEM), and their magnetic properties were characterized Vibrating Sample Magnetometer. The presence of \\
magnetite nanoparticles was indicated by the XRD spectral pattern and their SEM micrograph showed in the \\
nano ranged particles with an average diameter of 26.78 nm. Magnetic characteristic of magnetite nanoparticles \\
was indicated super paramagnetic properties with a saturation magnetic value of 90.01 emug ${ }^{-1}$. These magnetite \\
nanoparticles were used to remove the arsenic in the water by simple magnetic adsorption process and the \\
removal efficiency was found to be $96 \%$.
\end{tabular}

\section{INTRODUCTION}

Naturally occurring iron oxide $\left(\mathrm{Fe}_{3} \mathrm{O}_{4}\right)$ nanoparticle called as Magnetite was a contrary spinel having inimitable magnetic and electronic properties that were unswervingly related with the exceedingly fast electron exchange between the iron ions [1]. These iron atoms could be easily replaced by other divalent and trivalent metal ions. Aluminium, Magnesium, Manganese, and Titanium were the frequently occurring cations as impurities in naturally formed magnetite. This metal substitution in magnetite results in organized discrepancy in cell parameter, coercivity, Curie temperature, electrical resistivity, magneto crystalline anisotrophy, and saturation magnetization.

Magnetite has a wide range of applications in cancer treatment, magnetic resonance imaging, magnetic probes, pathogen detection, and as recording material [2]. Magnetite at nanosize has showed potential applications in medicine as diagnostic tools. Nanomagnetite were employed in either in vitro or in vivo applications, such as biomolecule immobilization and detection [3], contrast

*Corresponding Author

Gopal Samy Balakrishnan, Department of Biotechnology,

Roever College of Engineering and Technology, Perambalur, India.

E-mail: gopalsamy2k6@gmail.com agents in magnetic resonance imagining [4], gene transfer [5], cell separation [6], and purification [7]. They were also useful in target therapy as drug delivery system [3], and the heat generated in an alternating magnetic field was used for hyperthermia treatment [5].

The magnetite nanoparticle synthesis was of great importance owing to its technological applications. Magnetic nanoparticles were produced by many approaches, such as chemical precipitation [8], microbial synthesis [9], and size reduction through ball milling [10]. Consistent, recyclable, and harmless nanoparticles production was essentially important and microbial nanoparticle synthesis was one among them [11] and has interweaved microbial biotechnology and nanotechnology [12].

Inorganic materials in nano-scale dimensions were often produced by microorganisms. The microbial metal ion detoxification was done either by reduction or precipitation of soluble toxic inorganic ions to insoluble non-toxic metal nanoclusters. Mostly, extracellular metal nanoparticles production was used than Intracellular bioaccumulation as the former have more commercial applications in various fields.

Arsenite $\left(\mathrm{AsO}_{3}^{3-}\right)$ and arsenate $\left(\mathrm{AsO}_{4}^{3-}\right)$ were the natural forms of arsenic present in the groundwater. Arsenite and arsenate 
block ATP to ADP conversions by replacing phosphate groups permanently as these anions were similar to phosphite $\left(\mathrm{HPO}_{3}{ }^{2-}\right)$ and phosphate $\left(\mathrm{PO}_{4}^{3-}\right)$ ions [13]. Deep wells, ponds, rain water, and shallow wells are the sources of potable water for villages in underdeveloped countries. Health risks starting from microbial infections to diseases like arsenicosis caused by the high arsenic levels are associated with these sources. Switching to safer and deeper wells from many of these sources containing high arsenic levels has reduced the affected population [14].

Several treatment methods, such as adsorption, electrochemical reduction, ion exchange, nano filtration, precipitation, reverse osmosis, and solvent extraction are used to alleviate arsenic contamination [15]. But improved systems are needed as these technologies do not perform well in actual field trials [16]. Different sorbents, such as carbon from fly ash, coconut husk carbon, hybrid polymeric sorbent and iron-oxide-coated polymeric material [17], iron-coated activated carbon, Iron and iron-coated sand [18], and granular ferric hydroxides [19] have also been employed as adsorbents for As (III) adsorption. But, their application was limited owing to its high operation cost, sludge creation, and technical difficulties in their preparation. Naturally occurring ores and minerals, such as hematite and feldspar, kaolinite, magnetite, and maghemite were also employed for the arsenic adsorption [20,21].

The present study was aimed in microbial synthesis of magnetite nanoparticles using the fungal culture Fusarium oxysporum and employing these magnetite nanoparticles in arsenic removal.

\section{MATERIALS AND METHODS}

\subsection{Chemicals and Instruments Used}

The fungal culture $F$. oxysporum was procured from National Collection of Industrial Microorganisms (NCIM), CSIR-National Chemical Laboratory, Pune. All general reagents like distilled water and chemicals for media preparation in the present study were purchased from SD-Fine chemicals.

The FTIR Spectrophotometer analysis was done on RPilot IRTracer-100 Fourier Transform Infrared Spectrophotometer (Shimadzu, Japan). UV-Vis spectroscopy measurements were carried out using UV-1800 UV-VIS spectrophotometer (Shimadzu, Japan). X-ray diffraction (XRD) studies were performed on PANalytical's X'Pert PRO MRD. Scanning Electron Microscope (SEM) analysis was carried out on JSM-7610F Schottky Field Emission SEM (JEOL, Japan). The magnetic measurements were carried out by using 8600 Series Vibrating Sample Magnetometer (Lake Shore Cryotronics, USA).

\subsection{Sub Culture of $\boldsymbol{F}$. oxysporum}

The slant test tube of $F$. oxysporum procured from NCIM-NCL, Pune was sub-cultured in potato-dextrose-agar medium containing $200 \mathrm{~g}$ minced potato along with $20 \mathrm{~g}$ dextrose and $15 \mathrm{~g}$ agar in $1,000 \mathrm{ml}$ distilled water. The slant test tube was broken under sterile environment and the mycelia and spores of $F$. oxysporum were inoculated into plates by streak plate method. The inoculated Plates were incubated at $27^{\circ} \mathrm{C}$ at incubator for 7 days.

\subsection{Collection of Fungal Mat of $F$. oxysporum}

After 7 days of incubation, the fungal mat was collected from the culture broth by centrifuging at $3,500 \mathrm{rpm}$ for 20 minutes at $10^{\circ} \mathrm{C}$. Then, the mycelia were washed three times with sterile distilled water under sterile conditions.

\subsection{Synthesis of Magnetite Nanoparticles from $F$. oxysporum}

Fungal mat of $F$. oxysporum obtained from the potato dextrose broth was used for the synthesis of magnetite nanoparticles. $20 \mathrm{~g}$ wet weight of the fungal biomass of $F$. oxysporum was suspended in $100 \mathrm{ml}$ of a $0.001 \mathrm{M}$ aqueous solution of ferric chloride and magnetite natural ore on another $500 \mathrm{ml}$ Erlenmeyer flask. The flasks are kept on a shaker with $150 \mathrm{rpm}$ at $27^{\circ} \mathrm{C}$ for 3 days.

\subsection{Isolation of Magnetite Nanoparticles from Microbes}

After 3 days of incubation, the $F$. oxysporum fungal biomass was removed by centrifugation at 3,500 rpm. The culture supernatant containing nanoparticles was collected and centrifuged at $15,000 \mathrm{rpm}$ for 30 minutes to get the black pellets of magnetite nanoparticles. These magnetite nanoparticles were collected and washed thrice with $50 \mathrm{mM}$ sodium phosphate buffer ( $\mathrm{pH} 7.4$ ). The washed magnetite nanoparticles were allowed to air dry and the magnetite nanoparticles in the form of powder were collected.

\subsection{Characterization of Magnetite Nanoparticles}

The dried nanoparticles are characterized using the techniques described below.

\subsubsection{FTIR Spectrophotometer analysis}

The synthesized Magnetite nanoparticle powder was washed three times with $20 \mathrm{ml}$ of deionized water to get rid of the free proteins and enzymes that are not capping the nanoparticles. The nanoparticles were analyzed at a resolution of $1 \mathrm{~cm}^{-1}$ in the diffuse reflectance mode after drying it in room temperature and grinding with $\mathrm{KBr}$ pellets.

\subsubsection{UV-Vis spectroscopic analysis}

The formation of magnetite nanoparticles was confirmed by the determination of plasmon peak using UV-Vis spectroscopy. The reaction mixture absorbance indicating the reduction of nickel ions from ferric chloride was examined in the range of 360 to 800 $\mathrm{nm}$ and their maximum absorbance peak was calculated.

\subsubsection{XRD analysis}

XRD studies were performed on PANalytical's X'Pert PRO MRD having a copper X-ray source, a scintillation detector and in sample holders specifically made of a customized polymer to guard them from the ambient moisture. The XRD studies were performed without any treatment of samples.

\subsubsection{SEM analysis}

The nanoparticle suspension was dried into powder by critical point drier. About $1 \mathrm{mg}$ fine magnetite nanoparticle powder was 
used for the morphological analysis and the nanoparticle size was measured by SEM analysis.

\subsubsection{Ferromagnetism measurement}

Magnetometer was used to determine the magnetic properties of the synthesized nanoparticles. The applied magnetic field for the sample in the holder was slowly varied using magnetic field slider. Whenever the slider is stopped, $\mathrm{M}-\mathrm{H}$ graph corresponding to the field was plotted.

\subsection{Removal of Arsenic by Magnetite Nanoparticles}

\subsubsection{Preparation of adsorbent}

The magnetite nanoparticles synthesized from fungal method using $F$. oxysporum was used for arsenic removal from drinking water. In a sonication bath, $4 \mathrm{gl}^{-1}$ magnetite nanoparticles were dispersed in deionized water for 20 minutes.

\subsubsection{Water sample collection}

Arsenic rich groundwater was collected from shallow pond in Perampur, Tamil Nadu and used in the study. All water samples were immediately acidified after collection with $2 \% \mathrm{HNO}_{3}$ to avoid the precipitation of Fe-As compound and were kept in dark brown plastic bottles.

\subsubsection{Treatment with magnetite nanoparticles}

The Arsenic contaminated drinking water and nanoparticle mixture solution were held in a slowly rotating shaker at $40 \mathrm{rpm}$ speed for 6 hours. The mixtures following shaking were centrifuged for 30 minutes at 5,000 rpm. The precipitated solid samples were separated from supernatant and dried in vacuum desiccators. The dried arsenic adsorbed magnetite nanoparticles were kept in an airtight glass container to prevent any reaction with environment. The supernatant collected was filtered through $0.2 \mu \mathrm{m}$ Cellulose Acetate membrane filters and the fiat 5,000 rpm. The precipitated solid samples were separated from supernatant and dried in vacuum desiccators. The dried arsenic adsorbed magnetite na

\section{RESULTS AND DISCUSSION}

The iron oxide solution color was reliant on nanoparticle size and also specifies the chemical nature of the compound. This distinctive aspect is a prime quality in their chemical identification, and was employed in checking the compound purity [22]. The magnetite nanoparticles synthesized by $F$. oxysporum fungal biomass from ferric chloride and natural magnetite ore was jet black in color signifying it as a pure magnetite (Fig. 1). The formation of brownish-red products representing a mixture of iron oxides was the reaction end.

\subsection{FTIR Spectrophotometer Analysis}

The surface functional groups were characterised by Fourier Transform Infrared (FTIR) Spectroscopy method. The FTIR spectra of magnetite nanoparticles synthesized by $F$. oxysporum from $\mathrm{FeCl}_{2}$ and natural magnetite ore were shown in Figure 2. The presence of hydroxyl $(-\mathrm{OH})$ groups in the surfaces of magnetite

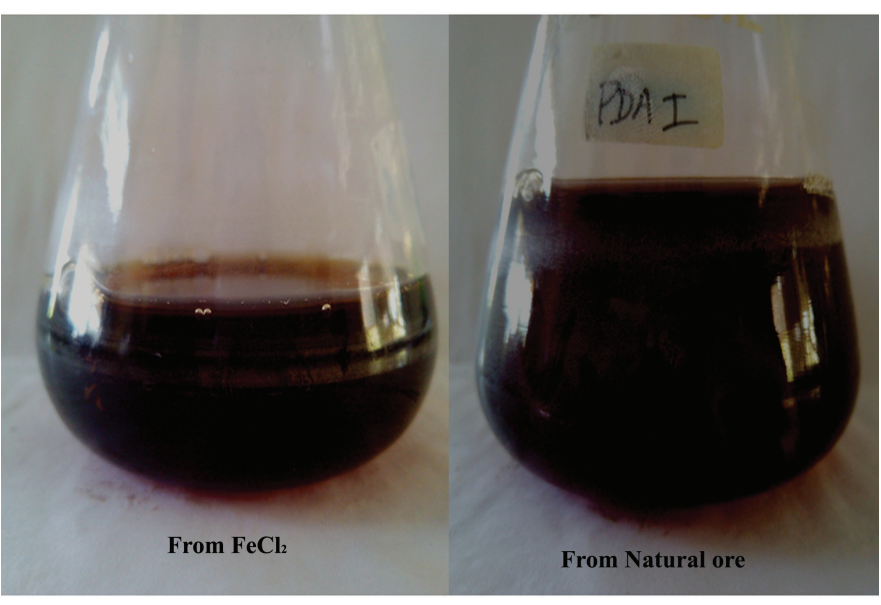

Figure 1: Colour changes of Magnetite nanoparticles synthesized.
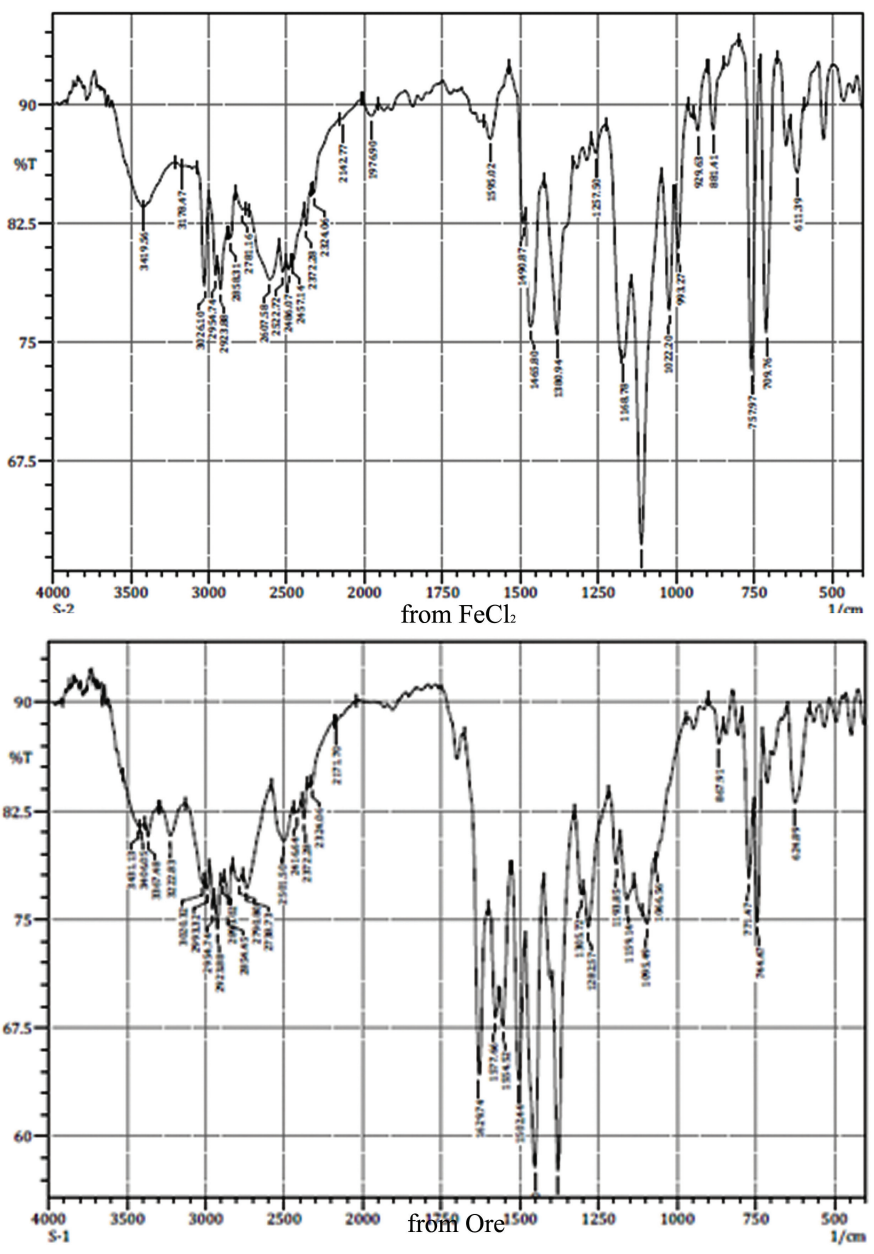

Figure 2: FTIR spectra of synthesized Magnetite nanoparticles.

nanoparticles were identified by the absorption band at a high wave number region of $3,414 \mathrm{~cm}^{-1}$. The tetrahedral and octahedral lattice site $\mathrm{Fe}-\mathrm{O}$ stretching vibration was obtained at $577 \mathrm{~cm}^{-1}$ [23]. The presence of broad absorption bands at 1,065 and 1,007 $\mathrm{cm}^{-1}$ indicated, respectively, the $\mathrm{C}-\mathrm{O}$ and $\mathrm{C}-\mathrm{OH}$ vibrations. A strong absorption area related to stretching and torsional 
vibration modes of magnetite were also observed further than $580 \mathrm{~cm}^{-1}$ and the band at $1,269 \mathrm{~cm}^{-1}$ represented the ferrophase complex vibrations. The deformation vibration of water molecule that was trapped onto the magnetite colloidal particles was seen at $1,633 \mathrm{~cm}^{-1}$. These values were similar to the FTIR analysis of chemically-produced magnetite nanoparticles [24].

\subsection{UV-Vis Spectrometry}

Figure 3 represents the UV-visible absorption spectra for magnetite nanoparticles produced by the fungal biomass of $F$. oxysporum. A surface plasmon resonance band of the magnetite nanoparticles around $415 \mathrm{~nm}$ showed a red-shift and peak broadening in the spectrum [25]. The reduction in intensity after the peak was caused by the oxidation of zero valent iron to iron oxide nanoparticles analogous to previous observations [26]. The reduction of $\mathrm{FeCl}_{3}$ into $\mathrm{Fe}_{3} \mathrm{O}_{4}$ was denoted by the maximum SPR band located around $420 \mathrm{~nm}$.

\subsection{XRD Analysis}

The XRD spectral patterns of the magnetite nanoparticles were shown in Figure 4. The XRD spectral peaks indicated that magnetite nanoparticles have a spherical structure and the XRD spectrum has no distinctive peak for impurities [27]. The average crystals

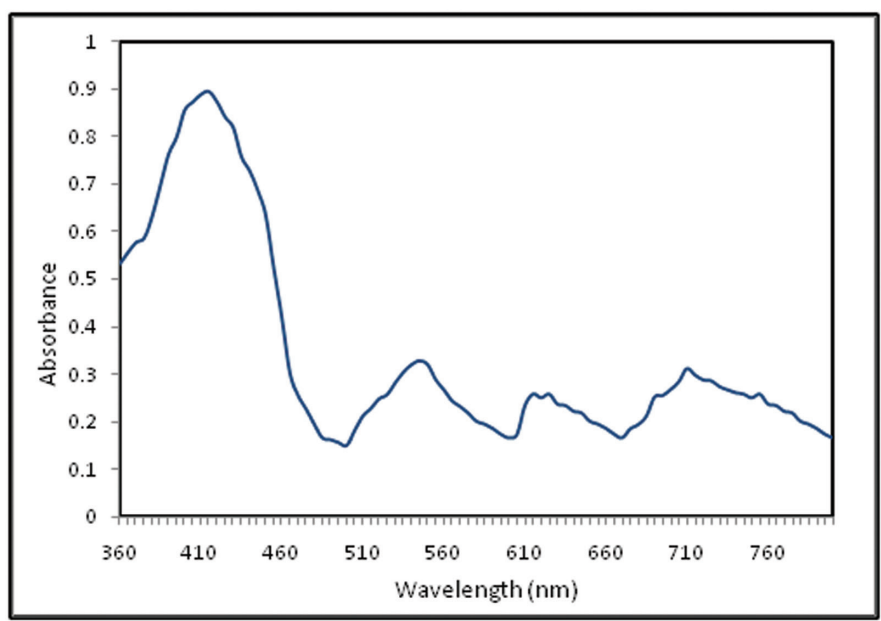

From $\mathrm{FeCl}_{2}$

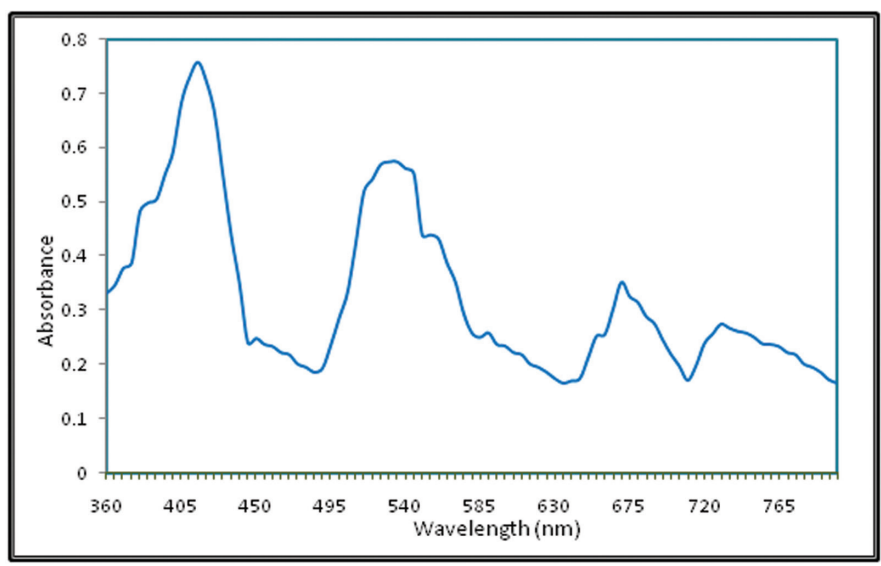

From Ore

Figure 3: UV spectra of synthesized Magnetite nanoparticles. size of the magnetite nanoparticle synthesized by $F$. oxysporum from $\mathrm{FeCl}_{2}$ and natural magnetite ore were, respectively, $31.29 \mathrm{~nm}$ and $39.52 \mathrm{~nm}$. The inverse spinel structure with a face-centered cubic phase of the produced magnetite nanoparticles were found from the relative intensity and position of the peaks in the XRD patterns that are analogous to the standard magnetite samples [28].

\subsection{SEM Analysis}

The direct observation of synthesized magnetite nanoparticles through high-resolution SEM revealed their size and morphology. The magnetite nanoparticle micrograph shown in Figure 5 clearly indicated that they were spherical, globular, and sometimes irregular in shape with a narrow size distribution in the range 27.54 to $81.22 \mathrm{~nm}$. The average particles size of the synthesized magnetite nanoparticles were, respectively, 46.98 and $52.49 \mathrm{~nm}$ for ferric chloride and natural magnetite ore. The sizes were similar to that of the Debye-Scherrer formula estimated size value and were also closer to those obtained chemically earlier [24].

\subsection{Vibrating Sample Magnetometer (VSM) Analysis}

The magnetization curve obtained by magnetometer for the synthesized magnetite nanoparticles were given in Figure 6. The saturation magnetization value of $45 \mathrm{emug}^{-1}$ for the synthesized
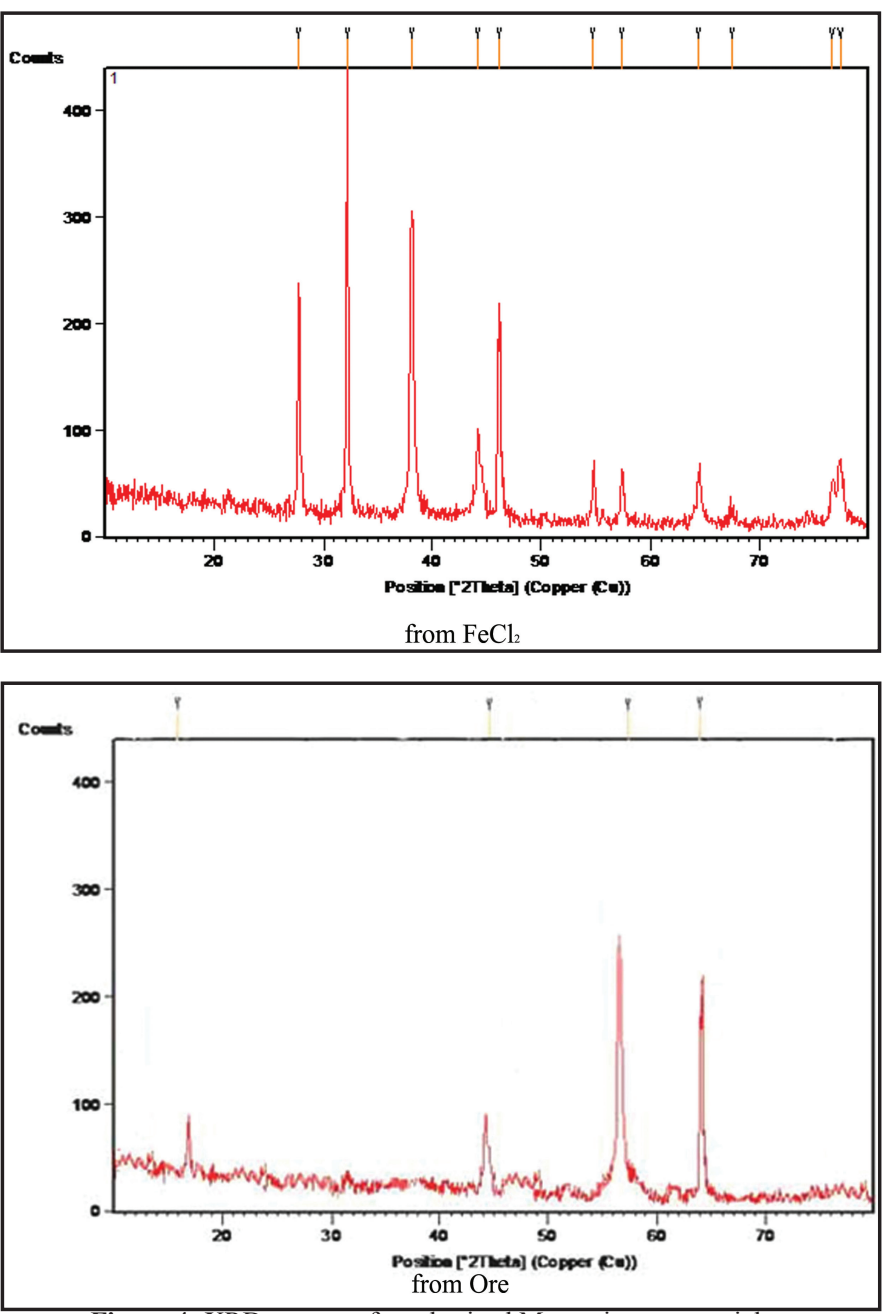

Figure 4: XRD spectra of synthesized Magnetite nanoparticles. 

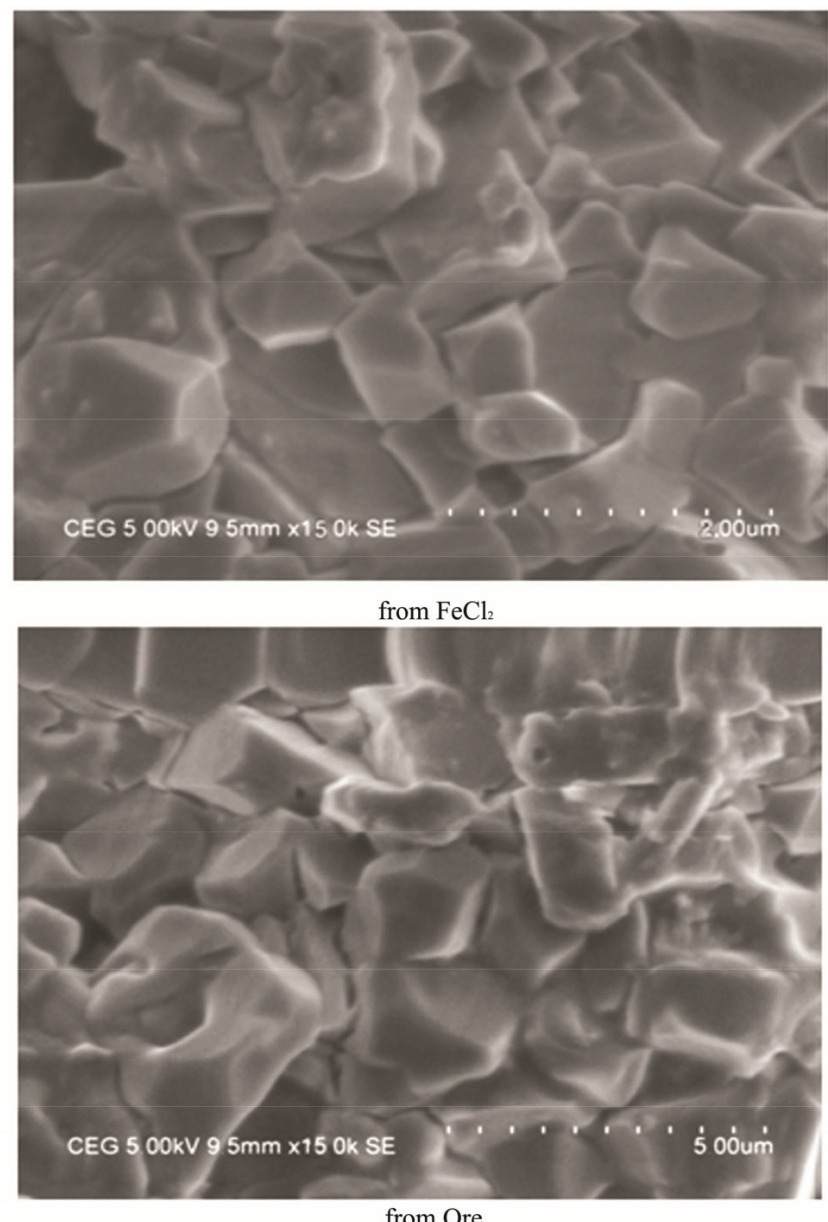

from Ore

Figure 5: SEM analysis of synthesized Magnetite nanoparticles.

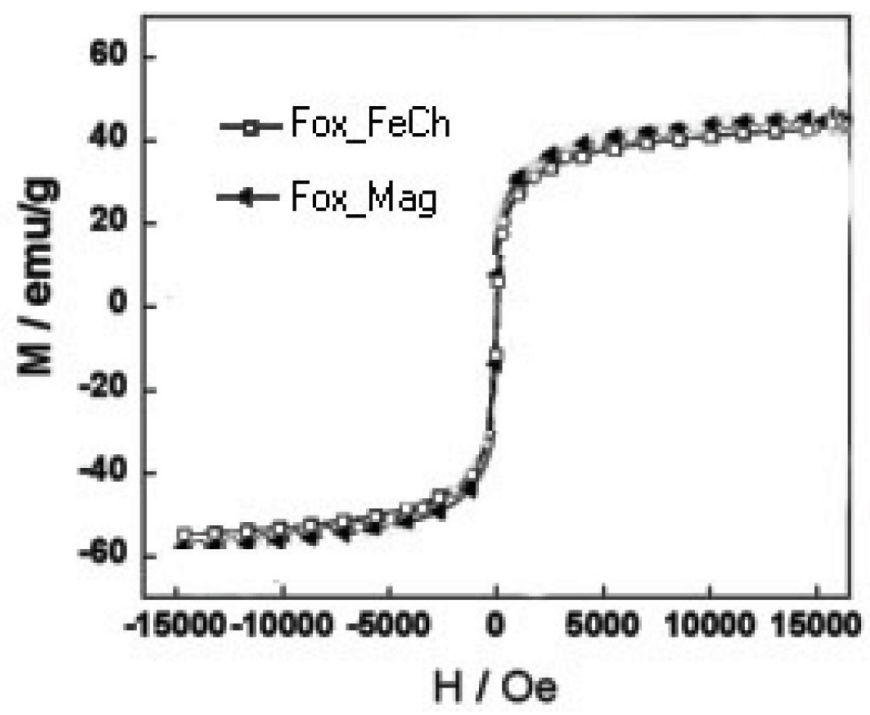

Figure 6: VSM analysis of synthesized Magnetite nanoparticles.

magnetite nanoparticle confirmed its super paramagnetic properties at room temperature. This exceptional magnetic property achieved was almost same to that of the magnetite nanoparticles produced

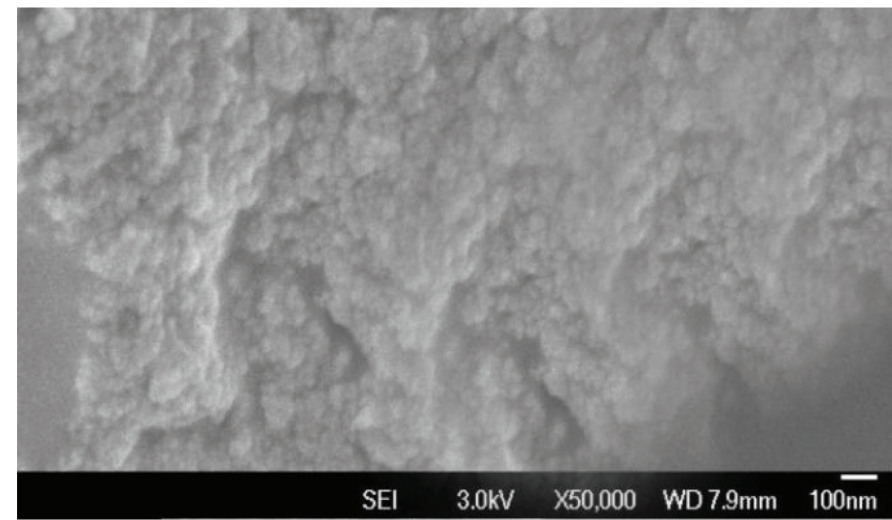

Figure 7: SEM of Magnetite nanoparticles after arsenic removal.

by chemical methods [24] and was vital for magnetic adsorption of arsenic from water.

\subsection{Arsenic Removal}

Based on the low average crystal size, magnetite nanoparticle synthesized by $F$. oxysporum from $\mathrm{FeCl}_{2}$ was used for the adsorption experiment. After adsorption experiments, the magnetite nanoparticles were collected and analyzed with SEM, and the supernatant was analyzed by Atomic Absorption Spectroscopy. The AAS analysis of the supernatant revealed that $91 \%$ of As (III) and $95.12 \% \mathrm{As}(\mathrm{V})$ were removed by the magnetite nanoparticles synthesized by F. oxysporum which was almost similar to the $95 \%$ removal by chemically synthesized magnetite nanoparticles [24]. The pore blockage in the SEM analysis confirmed that the space was filled with arsenic owing to the process of sorption (Fig. 7). The adsorption of arsenic by magnetite nanoparticles was clearly indicated in the SEM micrographs by the irregular shape and increased size of the magnetite nanoparticles.

\section{CONCLUSION}

The magnetite nanoparticles synthesized by the fungal culture of F. oxysporum was characterized for its physical appearance and magnetic properties. It was effective in removing arsenic from ground water as almost $95 \%$ of arsenic was adsorbed using an adsorbent dose of $4 \mathrm{gl}^{-1}$ at the optimum $\mathrm{pH}$ of 5.5. The results achieved so far was worthy as it contribute much to the future studies in implementing these nanoparticles for the arsenic removal from water making it safe for drinking purpose.

\section{CONFLICT OF INTEREST}

The authors declare that they do not have any conflicts of interest.

\section{FINANCIAL SUPPORT}

None.

\section{REFERENCES}

1. Kumar R, Sen S. Biogenic magnetite nanoparticle. Res J Pharm Biol Chem Sci 2013;4(3):1037-43.

2. Mohapatra M, Anand S. Synthesis and applications of nanostructured iron oxides / hydroxides a review. Int J Eng Sci Technol 2010;2(8): $127-46$. 
3. Tamer U, Gundogdu Y, Boyaci IH, Pekmez K. Synthesis of magnetic core-shell $\mathrm{Fe}_{3} \mathrm{O}_{4}-\mathrm{Au}$, nanoparticles for biomolecule immobilization and detection. J Nanopart Res 2010;12:1187-96.

4. Meng X, Seton HC, Lu LT, Prior IA, Thank NTK, Song B. Magnetic CoPt nanoparticles as MRI contrast agent for transplanted neural stem cells detection. Nanoscale 2011;3(3):977-84.

5. Laurent S, Dutz S, Häfeli UO, Mahmoudi M. Magnetic fluid hyperthermia: focus on super-paramagnetic iron oxide nanoparticles Adv Colloid Interface Sci 2011;10166(1-2):8-23.

6. Huang YF, Wang YF, Yan XP. Amine functionalized magnetic nanoparticles for rapid capture and removal of bacterial pathogens. Environ Sci Technol 2010;1544(20):7908-13.

7. Dong H, Huang J, Koepsel RR. Recyclable antibacterial magnetic nanoparticles grafted with quaternized poly (2- (dimethylamino) ethyl methacrylate) brushes. Biomacromolecules 2011;1112(4):1305-11.

8. Hyeon T. Chemical synthesis of magnetic nanoparticles. Chem Commun 2003;8:927-34.

9. Petrovsky E, Alcala MD, Criado JM, Grygar T, Kapicka A, Subrt J. Magnetic properties of magnetite prepared by ball-milling of hematite with iron. J Magn Magn Mater 2000;210:257-73.

10. Roh Y, Lauf RJ, McMillan AD, Zhang C, Rawn CJ, Bai J, et al. Microbial synthesis and the characterization of metal-substituted magnetites. Solid State Commun 2001;118:529-34.

11. Narayanan KB, Sakthivel N. Biological synthesis of metal nanoparticles by microbes. Adv Colloid Interface Sci 2010;156:1-13.

12. Li X, Xu H, Chen Z, Chen G. Biosynthesis of nanoparticles by microorganisms and their applications. J Nanomater 2011;Article ID 270974.

13. Jain CK, Ali I. Arsenic: occurrence, toxicity and speciation techniques. Water Res 2000;34(17):4304-12.

14. Ahmed MF, Ahuja S, Alauddin M, Hug SJ, Lloyd JR, Pfaff A, et al. Epidemiology-Ensuring safe drinking water in Bangladesh. Science 2006;314(5806):1687-8.

15. Mayo JT, Yavuz C, Yean S, Cong L, Shipley H, Yu W, et al. The effect of nanocrystalline magnetite size on arsenic removal. Sci Technol Adv Mater 2007;8:71-5.

16. Hossain MA, Sengupta MK, Ahamed S, Rahman MM, Mondal D, Lodh D, et al. Ineffectiveness and poor reliability of arsenic removal plants in West Bengal, India. Environ Sci Technol 2005;39(11): 4300-6.

17. Demarco MJ, Sengupta AK, Greenleaf JE. Arsenic removal using a polymeric/inorganic hybrid sorbent. Water Res 2003;37(1):164-76.
18. Petrusevski B, Boere J, Shahidullah SM, Sharma SK, Schippers JC. Adsorbent-based point-of-use system for arsenic removal in rural areas. J Water Supply Res Technol Aqua 2002;51:135-44.

19. Driehaus W, Jekel M. Granular ferric hydroxide-a new adsorbent for the removal of arsenic from natural water. J Water Supply Res Technol Aqua 1998;47:1-6.

20. Shipley HJ, Yean S, Kan AT, Tomson MB. Adsorption of arsenic to magnetite nanoparticles: Effect of particle concentration, ph, ionic strength, and temperature. Environ Toxicol Chem 2009;28(3):509-15.

21. Lim SF, Zheng YM, Chen JP. Organic arsenic adsorption onto a magnetic sorbent. Langmuir 2009;25(9):4973-8.

22. Cornell RM. The iron oxides: structure, properties, reactions, occurrences and uses. Wiley, New York, 2003.

23. Huang Z, Tang F. Preparation, structure, and magnetic properties of mesoporous magnetite hollow spheres. J Colloid Interface Sci $2005 ; 281: 432-6$.

24. Karthik R, Gopal Samy B, Jegatheesan K. Synthesis of magnetite nanoparticles for arsenic removal from ground water pond. Int J Pharmtech Res 2015;8(4):670-7.

25. Lyon JL, Fleming DA, Stone MB, Schiffer P, Williams ME. Synthesis of $\mathrm{Fe}$ oxide core/Au shell nanoparticles by iterative hydroxylamine seeding. Nano Lett 2004;4:719-23.

26. Guo L, Huang Q, Li X, Yang S. Iron nanoparticles: synthesis and applications in surface enhanced Raman scattering and electrocatalysis. Phys Chem Chem Phys 2001;3:1661-5.

27. Li GL, Jiang YR, Huang KL, Ding P, Yao II. Kinetics of adsorption of Sacharomyces cereviceae mandelated dehydrogenase on magnetic $\mathrm{Fe}_{3} \mathrm{O}_{4}$-chitosan nanoparticles. J Colloid Interface Sci 2008;A320:11-8

28. Wang B, Wei, Q, Qu S, Synthesis and characterization of uniform and crystalline magnetite nanoparticles via oxidation-precipitation and modified co-precipitation methods. Int $\mathrm{J}$ Electrochem Sci 2013;8:3786-93.

How to cite this article:

Balakrishnan GS, Rajendran K, Kalirajan J. Microbial synthesis of magnetite nanoparticles for arsenic removal. J Appl Biol Biotech 2020;8(03):070-075. DOI: 10.7324/ JABB.2020.803013 